\title{
Answer to the Letter to the Editor of B. Hu et al. concerning "Demographics, presentation and symptoms of patients with Klippel-Feil syndrome: analysis of a global patient-reported registry" by Nouri et al. [Eur Spine J; (2019) 28(10): 2257-2265]
}

\author{
Aria Nouri ${ }^{1}$
}

Received: 14 November 2019 / Accepted: 16 November 2019 / Published online: 26 November 2019

(c) Springer-Verlag GmbH Germany, part of Springer Nature 2019

Thank you for your interest in our paper. The purpose of our research was to investigate data of patients with KFS, a rare disease, to describe the demographics and identify possible associations with health problems based on the reported data-such an analysis is inherently explorative. While it is true that generally speaking Fisher's exact test is used when $20 \%$ of cells with an expected value of less than 5 are present in a $2 \times 2$ comparison, this test has also been considered to be conservative [1]. The concern that not having used this test has led to inappropriate conclusions is incorrect.

Four comparisons in Table 4 would qualify for Fisher's exact test, and the changes to $p$ values would be the following: Missing Ribs $p=0.054$ (from $p=0.046$ ); Tachycardia $p=0.091$ (from $p=0.071$ ); Restrictive Lung Disease $p=0.091$ (from $p=0.071$ ); and Limited Range of Motion in Spine $p=0.038$ (from $p=0.022$ ). Using a Chi-square test or Fisher's exact test for Missing Ribs leads to a $p$ value that rounds to 0.05 -borderline significant in either case. The comparison of the other three factors also does not change our conclusion that two of these factors were trends, in other words, not significant, but with a $p$ value $<0.10$, and one factor was statistically significant with a $p$ value $<0.05$.
The conclusions in the paper remain sound for three principle reasons: (1) No material differences are observed with the application of Fisher's exact tests in the present analysis. (2) Conclusions were balanced with caution, and it was clearly stated in the paper that Missing Ribs may be associated with multi-level fusions. (3) The limitations section of the paper clearly indicates that findings may be prone to type 1 error, highlighting the exploratory nature of our results and need for confirmatory research to substantiate the findings.

\section{Compliance with Ethical Standards}

Conflict of interest Dr. Nouri has no conflicts of interest to disclose.

\section{Reference}

1. Ludbrook J (2008) Analysis of $2 \times 2$ tables of frequencies: matching test to experimental design. Int J Epidemiol 37:1430-1435

Publisher's Note Springer Nature remains neutral with regard to jurisdictional claims in published maps and institutional affiliations.
Aria Nouri

arianouri9@gmail.com

1 Department of Neurosurgery, Geneva University Hospitals, Geneva, Switzerland 